\title{
Evaluation of Remineralization Potential of ACTIVA Bioactive Restorative Material Versus Resin Modified Glass Ionomer in Restoration of Premolars: In Vitro Study
}

\section{Sally Mohamed Mosallam*, Randa Abdel-Gawad, Fatma El- Shehaby and Marwa Elchaghaby}

Department of Pediatric Dentistry and Dental Public Health, Cairo University,

Egypt

*Corresponding Author: Sally Mohamed Mosallam, Department of Pediatric Dentistry and Dental Public Health, Cairo University, Egypt.
Received: April 06, 2021

Published: April 30, 2021

(C) All rights are reserved by Sally Mohamed Mosallam., et al.

\section{Abstract}

Aim or Purpose: This study aims to evaluate the remineralization potential of ACTIVA Bioactive restorative material compared to light-cured resin-reinforced glass ionomer restorative.

Materials and Methods: A standardized Class V cavity was prepared in forty-two extracted sound human premolars. The mineral content of the teeth was measured before and after demineralization, then the demineralized teeth were left as a negative control (Group I) or restored with either ACTIVA ${ }^{\mathrm{TM}}$ Bioactive restorative material (Group II) or light-cured resin-reinforced glass ionomer restorative (Group III). The teeth were stored in artificial saliva for $23 \mathrm{~h}$ and $1 \mathrm{~h}$ in lactic acid solution at $37^{\circ} \mathrm{C}$ to simulate the oral environment and the acid challenges occurring intraorally. The mineral content was evaluated using energy dispersive x-ray and scanning electron microscope (EDX/SEM) in each group after 24h, 1 month later and after three months.

Results: A significant statistical difference in fluoride and calcium release between the two groups was observed. Light-cured resinreinforced glass ionomer restoration showed higher release of fluoride compared to ACTIVA ${ }^{\mathrm{TM}}$ Bioactive restorative material while ACTIVA $^{\mathrm{TM}}$ Bioactive restorative material showed higher release of calcium compared to the light-cured resin-reinforced glass ionomer. There was no statistical difference in phosphorus release between the two tested materials. Phosphorus content was relatively similar in both groups.

Conclusion: Resin-modified glass ionomer materials exhibit greater fluoride release than resin-based materials ACTIVIA BioactiveRestorative with the highest fluoride release taking place during the first 24 hours. ACTIVA Bioactive-Restorative could be considered a suitable class $\mathrm{V}$ restoration in high-caries risk patients.

Keywords: Remineralization Potential; ACTIVA Bioactive Restorative Material; Resin Modified Glass Ionomer

\section{Introduction}

Dental caries remains one of the most prevalent chronic infectious diseases of childhood in both developed and developing countries. The earlier approaches for caries management were surgical. Placing a filling in a diseased tooth only restores the damage caused by the disease but never treats dental caries [19].
Fluoride-containing restorative materials gained great attention over the last two decades as a means of protection against recurrent caries. Fluoride increases tooth resistance to caries through different protective mechanisms. There are several fluoride-containing hybrid restorative materials available in the market including resin-modified glass ionomers, compomers, giomers and bioactive resin composites [7]. 
Hybrid materials combining the technologies of glass-ionomer and composites were developed to overcome the disadvantages of glass ionomer cement, among them, were resin-modified glass ionomer that provides an initial burst of fluoride release and better mechanical properties than conventional glass ionomer [3].

The main purpose of modification in conventional glass ionomer restorations was to improve the performance of these materials without affecting the fluoride release characteristics. Resin or acid modification aimed to control the moisture sensitivity during the setting reaction and to improve the strength in the initial stages [25].

The mechanical properties of resin-modified glass ionomers show greater flexural strength and flexural fatigue compared with conventional glass ionomers, however, their resistance to wear in areas of mechanical stress still low also, it has low compressive strength compared to other restorative materials that can be used in the stress-bearing areas. This could be attributed to the microstructure, porosity, content, molecular weight of polyacids and resins, size, content, and distribution of inorganic particles or fillers which may harm the compressive strength of the material and so in their clinical performance [1,207].

In the last years, there has been an increasing interest in the application of bioactive materials in the dental field in an attempt to remineralize affected dentin along with fluoride release and recharge capacity. One of the leading materials in this class is ACTI$\mathrm{VA}^{\mathrm{TM}}$ restorative material $[4,10]$.

ACTIVA $^{\mathrm{TM}}$ restorative material bioactive restorative material was developed in an attempt to overcome the disadvantages of glass ionomer (GIC) and resin-based composite (RBC) and to combine their advantages in one restorative material [5].

\section{Aim of the Study}

This study aims to evaluate the remineralization potential of two commercially available fluoride-containing restorative materials: ACTIVATM Bioactive restorative material and light-cured resin-reinforced glass ionomer restorative in human premolars using EDX/SEM elemental analysis.

\section{Materials and Methods}

Study design

The present study is an in-vitro experimental study carried out to assess the remineralization potential of ACTIVATM Bioactive restorative material compared to light-cured resin-reinforced glass ionomer restorative in premolars using EDX/SEM elemental analysis.

Study setting:

42 samples of sound premolars extracted for orthodontic treatment purpose were collected from Orthodontic Department, Faculty of Dentistry, Cairo University.

The premolars enrolled in this study were selected according to Prabhakar., et al.:

- Freshly extracted sound premolars without any previous restoration or fractures.

- Extracted Premolars free of cracks and developmental disturbances.

- $\quad$ Extracted Premolars free of stains.

- Extracted Premolars of a normal anatomical structure.

\section{Ethical approval}

Ethical approval was obtained from the Research Ethics Committee, Faculty of Dentistry, Cairo University (no. 19513).

\section{Sample preparation}

After extraction, the teeth were thoroughly cleaned using a slurry of fluoride-free pumice, on a prophylactic brush, mounted on a low-speed contra angled handpiece, then rinsed using a triple air/ water syringe. Standardized non - beveled buccal class V cavities were prepared following the cavity preparation guidelines $(3 \mathrm{~mm}$ wide, $2 \mathrm{~mm}$ high, $1.5 \mathrm{~mm}$ deep) in the cervical third of each tooth. Using a standardized No: 108008, $0.8 \mathrm{~mm}$, Horico ${ }^{\circledR}$ diamond bur mounted at high speed with air/water cooled contraangled handpiece. For standardization, William's graduated probe was used to measure the dimensions of the cavity. Prepared teeth were cleaned with air/water mixture from a triple air/water syringe and dried with oil-free compressed air for 15 seconds.

Cavities were acid-etched with $37 \%$ orthophosphoric acid for 15 seconds to create demineralization, then were cleaned in distilled water for 10 minutes to remove any debris. Then stored at $4^{\circ} \mathrm{C}$ in phosphate-buffered saline (PBS, $\mathrm{pH} 7.4$ ) before use [13].

Assignment of teeth

After that, teeth were randomly subdivided into three experi- 
mental groups (14 teeth per each group), one group was used as a negative control (C), the two other groups were subdivided according to the type of material used (R), where R1; the cavities restored with Bioactive restorative material (ACTIVA ${ }^{\mathrm{TM}}$ ) and R2; the cavities restored with Light cured resin-reinforced glass ionomer restorative (FUJI II ${ }^{\mathrm{TM}} \mathrm{GC}$ ).

\section{Storage of the samples}

After the completion of the restorative procedures, teeth included in the study were stored in a labeled plastic vial containing $1 \mathrm{~mL}$ of artificial saliva of $\mathrm{pH}=7$, to approximate the physiologic values for saliva in the oral cavity. Every day, each sample of the mentioned two groups was immersed in $1 \mathrm{~mL}$ of fresh artificial saliva for 23 hours and then in $1 \mathrm{~mL}$ of the lactic acid solution for $1 \mathrm{~h}$ at $37^{\circ} \mathrm{C}$ using an incubator, this technique was repeated for 12 weeks. The 1-h duration in the lactic acid solution simulates the accumulated acid challenge times in a 24 -h period orally [13].

\section{Slicing and laboratory evaluation}

The teeth were sectioned buccolingually into two halves in a vertical plane parallel to the long axis of the tooth. The sectioning was performed using a micro saw (Iso Met 4000). Then the roots of the teeth were cut horizontally, figure to allow the proper adjustment of the samples into the EDX/SEM apparatus.

Energy Dispersive X-ray (EDX) was employed as a chemical microanalysis technique to characterize the various samples at baseline, after demineralization and after application of restorative materials to determine the degree of remineralization at regular time intervals ( 24 hours, four weeks and twelve weeks) and comparing them with the controls.

The degree of remineralization was assessed by measuring the net intensity of phosphorus (P) calcium (Ca) and fluoride (F) in the restored samples after immersion in the artificial saliva solution and lactic acid solution. Calcium hydroxyapatite $\left[\mathrm{Ca}_{10}\left(\mathrm{PO}_{4}\right)_{6}(\mathrm{OH})_{2}\right]$ is the mineral phase of dentin and thus a recorded increase in the levels of $\mathrm{P}$ and Ca suggested remineralization of dentin.

\section{Statistical analysis}

Statistical analysis was performed using SPSS $20^{\circ} 1$, Graph Pad Prism $^{\circ} 2$, and Microsoft Excel 20163. Data were represented as mean and standard deviation and the $p$-value was set at $<0.05$. Data were explored for normality by using Shapiro Wilk and Kol-
mogorov-Smirnov normality test. The comparison between the two groups at different follow-up periods was performed by the Independent T-test and the comparison between the follow-up periods was performed by the One Way ANOVA test followed by Tukey's Post Hoc test for multiple comparisons.

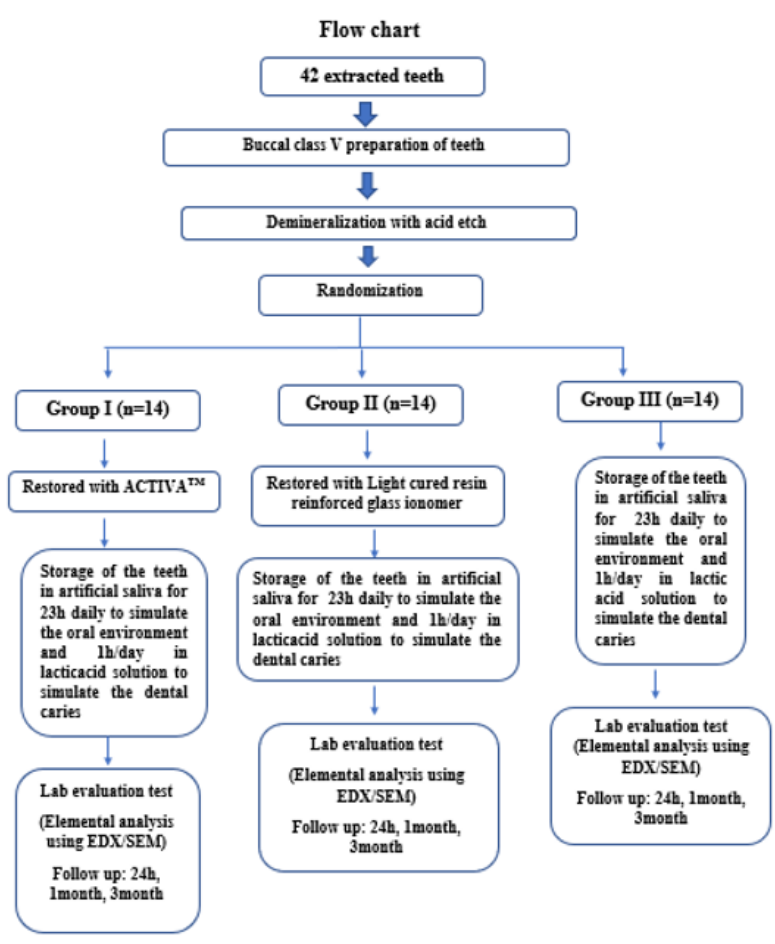

Flow chart

\section{Results}

The remineralization ability of two different fluoride-releasing restorative materials (Group I: Bioactive restorative material (ACTIVA $^{\mathrm{TM}}$ ) and Group II: Light cured resin-modified glass ionomer restorative) was evaluated using energy dispersive x-ray under scanning electron microscope (SEM-EDX analysis).

\section{Fluoride weight $\%$}

The mean and standard deviation (SD) for the net intensity of fluoride content measured as wt.\% shown in table 1, the resin modified glass ionomer showed the highest fluoride release throughout the study with the peak during the $1^{\text {st }} 24$ hours. Com- 
Evaluation of Remineralization Potential of ACTIVA Bioactive Restorative Material Versus Resin Modified Glass Ionomer in Restoration of Premolars: In Vitro Study

parison between group I - restoration I (Bioactive restorative material ACTIVA), group II - restoration II (Light cured resin reinforced glass ionomer restorative FUJI II) and group III (control), was performed by One Way ANOVA test which revealed significant difference between them $(p<0.05)$ regarding T2, T3, T4, followed by Tukey`s Post Hok test for multiple comparisons which revealed significant difference between them in means with different superscript letters as $\mathrm{p}<0.05$ (between group I, II and III at T2,T3 and T4), while revealed insignificant difference in means with the same superscript letters as $\mathrm{p}>0.05$ (between group I, II and III at T0 and T1) as presented in table 1.

\begin{tabular}{|l|c|c|c|c|c|c|c|}
\hline \multirow{2}{*}{$\begin{array}{l}\text { Fluo- } \\
\text { ride }\end{array}$} & \multicolumn{2}{|c|}{ Group I } & \multicolumn{2}{c|}{ Group II } & \multicolumn{2}{c|}{ Group III } & \multirow{2}{*}{} \\
\cline { 2 - 7 } & M & SD & M & SD & M & SD & \\
\hline T0 & $1.12 \mathrm{a}$ & 0.21 & $1.03 \mathrm{a}$ & 0.18 & $1.12 \mathrm{a}$ & 0.10 & 0.28 \\
\hline T1 & $0.51 \mathrm{a}$ & 0.14 & $0.52 \mathrm{a}$ & 0.16 & $0.50 \mathrm{a}$ & 0.14 & 0.93 \\
\hline T2 & $27.78 \mathrm{~b}$ & 4.21 & $31.11 \mathrm{c}$ & 4.13 & $0.50 \mathrm{a}$ & 0.14 & $0.0001^{*}$ \\
\hline T3 & $24.74 \mathrm{~b}$ & 2.39 & $26.93 \mathrm{c}$ & 2.51 & $0.98 \mathrm{a}$ & 0.34 & $0.0001^{*}$ \\
\hline T4 & $24.71 \mathrm{~b}$ & 1.52 & $25.91 \mathrm{c}$ & 1.42 & $0.66 \mathrm{a}$ & 0.30 & $0.0001^{*}$ \\
\hline
\end{tabular}

Table 1: Comparison between the three groups regarding the Fluoride wt \%.

\section{Phosphorus weight \%}

The mean and standard deviation (SD) the mean and standard deviation (SD) for the net intensity of phosphorus content measured as wt.\% shown in table 2, Comparison between group I - restoration I (Bioactive restorative material ACTIVA), group II - restoration II (Light cured resin reinforced glass ionomer restorative FUJI II) and group III (control), was performed by One Way ANOVA test which revealed significant difference between them $(\mathrm{P}<0.05)$ regarding T2, T3, T4, followed by Tukey`s Post Hok test for multiple comparisons which revealed significant difference between them in means with different superscript letters as $\mathrm{P}<0.05$ (between group I, II and III at T2, T3 - between group I and II at T4- between group I and III at T4), while revealed insignificant difference in means with the same superscript letters as $\mathrm{P}>0.05$ (between group I, II and III at T0 and T1 - between group II and III at T4) as presented in table 2 .

\begin{tabular}{|l|c|c|c|c|c|c|c|}
\hline \multirow{2}{*}{$\begin{array}{l}\text { Pho- } \\
\text { sph- } \\
\text { orus }\end{array}$} & \multicolumn{2}{|c|}{ Group I } & \multicolumn{2}{c|}{ Group II } & \multicolumn{2}{c|}{ Group III } & \multirow{2}{*}{$\begin{array}{c}\text { p } \\
\text { value }\end{array}$} \\
\cline { 2 - 7 } & M & SD & M & SD & M & SD & \\
\hline T0 & $31.12 \mathrm{a}$ & 1.74 & $31.04 \mathrm{a}$ & 1.77 & $31.05 \mathrm{a}$ & 1.78 & 0.91 \\
\hline T1 & $10.19 \mathrm{a}$ & 1.01 & $10.21 \mathrm{a}$ & 1.15 & $10.20 \mathrm{a}$ & 1.00 & 0.83 \\
\hline T2 & $26.55 \mathrm{~b}$ & 1.68 & $21.82 \mathrm{c}$ & 1.23 & $10.20 \mathrm{a}$ & 1.00 & $0.001^{*}$ \\
\hline T3 & $27.34 \mathrm{~b}$ & 1.01 & $24.51 \mathrm{c}$ & 1.42 & $9.49 \mathrm{a}$ & 0.98 & $0.001^{*}$ \\
\hline T4 & $27.32 \mathrm{~b}$ & 1.12 & $27.29 \mathrm{~b}$ & 1.84 & $11.49 \mathrm{a}$ & 1.46 & $0.001^{*}$ \\
\hline
\end{tabular}

Table 2: Comparison between the three groups regarding phosphorus wt \%.

\section{Calcium weight \%}

The mean and standard deviation (SD) for the net intensity of calcium content measured as wt.\% shown in table 3. Comparison between group I - restoration I (Bioactive restorative material ACTIVA), group II - restoration II (Light cured resin reinforced glass ionomer restorative FUJI II) and group III (control), was performed by One Way ANOVA test which revealed significant difference between them $(\mathrm{P}<0.05)$ regarding $\mathrm{T} 2, \mathrm{~T} 3, \mathrm{~T} 4$, followed by Tukey`s Post Hok test for multiple comparisons which revealed significant difference between them in means with different superscript letters as $\mathrm{P}<0.05$ (between group I, II and III at T2, T3 and T4), while revealed insignificant difference in means with the same superscript letters as P > 0.05 (between group I, II and III at T0 and T1) as presented in table 3 .

\begin{tabular}{|l|c|c|c|c|c|c|c|}
\hline \multirow{2}{*}{$\begin{array}{l}\text { Cal- } \\
\text { ci- } \\
\text { um }\end{array}$} & \multicolumn{2}{|c|}{ Group I } & \multicolumn{2}{c|}{ Group II } & \multicolumn{2}{c|}{ Group III } & \\
\cline { 2 - 8 } & M & SD & M & SD & M & SD & \\
\hline T0 & $63.48 \mathrm{a}$ & 3.55 & $63.53 \mathrm{a}$ & 3.14 & $63.49 \mathrm{a}$ & 3.55 & 0.93 \\
\hline T1 & $21.82 \mathrm{a}$ & 3.02 & $21.78 \mathrm{a}$ & 2.89 & $21.82 \mathrm{a}$ & 3.00 & 0.92 \\
\hline T2 & $50.75 \mathrm{~b}$ & 5.39 & $38.26 \mathrm{c}$ & 4.42 & $21.82 \mathrm{a}$ & 3.00 & $0.001^{*}$ \\
\hline T3 & $52.17 \mathrm{~b}$ & 2.89 & $44.73 \mathrm{c}$ & 3.12 & $21.02 \mathrm{a}$ & 4.10 & $0.001^{*}$ \\
\hline T4 & $54.74 \mathrm{~b}$ & 3.12 & $44.87 \mathrm{c}$ & 3.33 & $24.94 \mathrm{a}$ & 3.06 & $0.001^{*}$ \\
\hline
\end{tabular}

Table 3: Comparison between the three groups regarding calcium wt \%. 


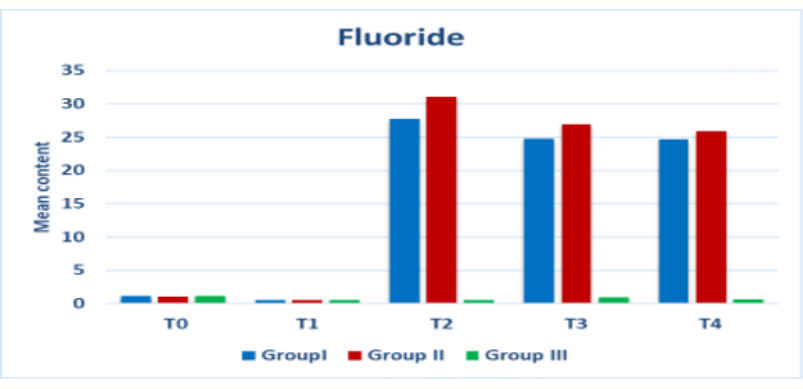

Figure 1: Bar chart presenting fluoride of R1 and R2 and C regarding all follow up periods.

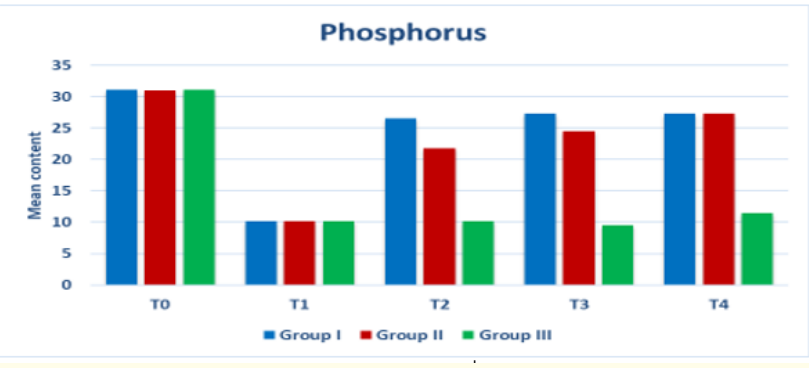

Figure 2: Bar chart presenting phosphorus of R1 and R2 and C regarding all follow up periods.

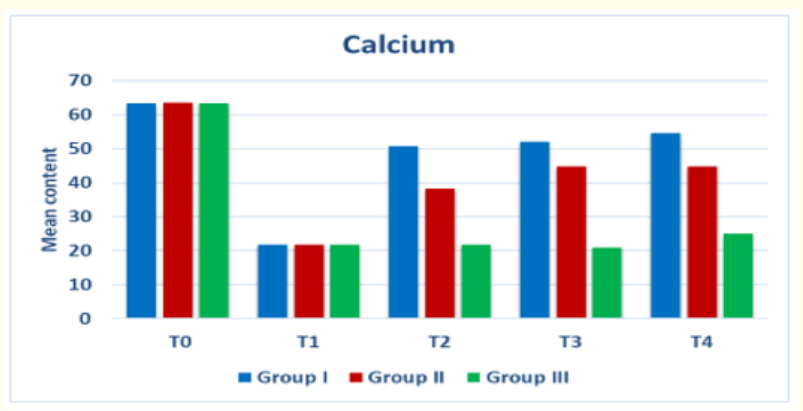

Figure 3: Bar chart presenting Calcium of R1 and R2 and C regarding all follow up periods.

\section{Discussion}

It is essential to evaluate the performance of newly produced glass ionomers in the laboratory before these materials can be tested in clinical studies. Therefore, the primary objective of this study was to assess and compare in-vitro the fluoride, phosphorus and calcium releasing capacity of two different bioactive restorative materials: ACTIVA ${ }^{\mathrm{TM}}$ Bioactive Restorative Material and Light cured resin-modified glass ionomer (FUJI II).

In this study, only sound teeth with normal anatomical structure, free of cracks, developmental disturbances, stains, and without any restoration or fractures were used, to provide standardization and avoid any confounding factor [18].

The samples were assigned to the corresponding groups using simple randomization with the help of the sealed envelope method. Randomization was done to eliminate selection bias; groups balance with respect to known and unknown confounding or prognostic variables and formation of the basis for statistical tests by the assumption of a free statistical analysis as denoted by [23]. In the present study, the operator was not blinded to the groups' allocation as both interventions had different clinical performance and shades. However, the statistician was completely blinded to the treatment groups [9].

To standardize the procedures, the cavity dimensions were kept similar for each tooth ( $3 \mathrm{~mm}$ wide, $2 \mathrm{~mm}$ high, $1.5 \mathrm{~mm}$ deep) and all teeth were prepared by a single operator [18]. All samples were demineralized for 15 seconds and rinsed with water to create a 5 $\mathrm{mm}$ thick layer of mineral-free collagen matrix on the surface of the mineralized dentine base [13].

The aging process used in this study was $\mathrm{pH}$ cycling as dental restorations are subjected to constant and extreme $\mathrm{pH}$ changes in the oral environment, with large fluctuations in the $\mathrm{pH}$. Therefore, $\mathrm{pH}$ cycling is an important procedure for testing the remineralization ability of restorative material [13]. The storing plastic vials were because it was reported that glass containers can react with fluoride absorption.

The mineral content was measured before the material application to have a baseline measurement, $24 \mathrm{hrs}$ after material application because the mineral release is highest after 24 hours, one month later and after three months to monitor the mineral release over time [18].

Many methods have been employed to estimate the mineral content such as spectrophotometry, ion chromatography, capillary electrophoresis, and energy dispersive x-ray with scanning 
electron microscope [21]. Mineral content measurements in this study were performed using energy dispersive $\mathrm{x}$-ray with scanning electron microscope as it gives an accurate and direct estimate of the minerals present along with the tooth/restoration interfaces at pulpal regions [2]. EDX is a reproducible, reliable, and precise technique to identify and quantify major components present in a material. The identification of constituents in materials leads to an understanding of its various physical, biological, chemical, and mechanical properties. However, EDX has limitations for the precise detection of low molecular weight elements such as carbon, hydrogen and oxygen. The proportion of ionizing episodes that result in the emission of X-rays decreases with elements of smaller atomic number [21].

In the present study, environmental temperature, the type and $\mathrm{pH}$ of the storage medium, the experimental design, and the analytic method were standardized for the two materials. Fluoride release from restorative materials is a complex process and is affected by intrinsic and extrinsic factors. Intrinsic factors include formulation, powder/liquid ratio, specimen geometry, temperature, mixing time, solubility or porosity of the material, surface treatment, and finishing. Extrinsic factors include the type and $\mathrm{pH}$ of storage medium, experimental design, environmental temperature, and analytical methods [11].

Regarding the control group there was minimal increase in the mineral content mainly calcium and phosphorus respectively, this may be attributed to the uptake of minerals ( $\mathrm{Ca}$ and $\mathrm{P}$ ) from the artificial saliva as an attempt for natural repair process, however, this mineral uptake was insufficient alone because of the daily acid challenge (pH-cycling) performed in this study [13].

Regarding the fluoride release, ACTIVA showed relatively high release after 24 hours which markedly declined over the first month. In the third-month fluoride release became relatively constant. These findings are in agreement with [15] who evaluated the release of fluoride ions from different fluoride-releasing materials and found that ACTIVA can maintain constant release of fluoride over 21 days without recharging.

According to Elbahrawy and Attia [6], ACTIVA showed a relatively constant fluoride release for 28 days and a small amount of fluoride release and recharge. This constant release may be attributed to the higher resin filler content (Urethane dimethacrylate monomers) which limits ion exchange with the external environment. Also, this was related to the slower dissolution of glass particles through the pores of the restorations with time. During the maturation period, bulk fluoride release occurs as a consequence of contact between the materials with the storage medium [16].

Concerning the phosphorus release, ACTIVA $^{\mathrm{TM}}$ showed approximately constant phosphorus release throughout the study period. ACTIVA $^{\mathrm{TM}}$ releases a certain amount of Phosphate, especially in a low $\mathrm{pH}$ environment. The cumulative amount of Phosphate release from ACTIVA $^{\mathrm{TM}}$ in 7 days period is around $300 \mathrm{mcg} / \mathrm{g}$ in $\mathrm{pH} 4$, and $100 \mathrm{mcg} / \mathrm{g}$ in $\mathrm{pH}$ 7, indicating that ACTIVA exhibits different behavior according to the acidity of the environment [12].

Regarding calcium release, ACTIVA $^{\mathrm{TM}}$ showed an increasing level of release throughout the study period that was higher than that of resin-modified glass ionomer. This may be due to the acid-catalyzed hydrolysis of the Al-O-Si bonds in the fluoro alumino-silicate ionomer glasses present in ACTIVA $^{\mathrm{TM}}$ which take place at low $\mathrm{pH}$ [24].

This finding is in agreement with Morrow., et al. [15] who found that ACTIVA ${ }^{\mathrm{TM}}$ released the highest amount of $\mathrm{Ca}^{2+}$ when compared to Filtek Supreme, Ketac Fil, and Equia Forte over 21 days, they found that ACTIVA ${ }^{\mathrm{TM}}$ has the ability to release calcium ions of an amount of $0.72 \mu \mathrm{g} / \mathrm{mm}^{2}$ within 7 days.

The previous finding may be explained by the fact that ACTI$\mathrm{VA}^{\mathrm{TM}}$ responds to $\mathrm{pH}$ cycles and plays an active role in maintaining oral health with the release and recharge of significant amounts of calcium, phosphate, and fluoride. These mineral components stimulate the formation of a protective/connective-apatite layer and a natural bonded-seal at the material-tooth interface [14].

Regarding resin-modified glass ionomer restoration, RMGICs showed the highest release of fluoride during the $1^{\text {st }}$ day. This may be attributed to the initial "burst" of fluoride release from the glass particles. The burst release is attributed to the reaction of the polyalkenoic acid with the fluoride-containing glass particles during the setting reaction and also to the rapid dissolution of fluoride from the outer surface into the solution [6].

Later the fluoride release decreased to a continuous plateau till the end of the study. The slower release of fluoride during subse- 
quent days has been attributed to the slower dissolution of glass particles through cement pores and fractures. Bulk fluoride diffusion occurs during the maturation period as a consequence of contact between the GIC material with the storage medium [11].

These results are in agreement with different in-vitro studies that have also shown higher fluoride release in the first two days of the study followed by decreased release after the first weak. RMGICs show an initial burst of fluoride release followed by a marked decrease of release throughout the next days $[1,8]$.

Regarding the comparison between the two tested materials, after reviewing the literature, only three studies compared the fluoride release between ACTIVA $^{\mathrm{TM}}$ and resin-modified glass ionomer restoration $[7,15,22]$. There were no studies in the literature comparing the calcium and phosphorus release between the two materials used in this study.

The results of $[7,22]$ were in agreement with the current study results regarding the higher initial release of fluoride with RMGICs which was followed by a marked decrease and the relatively constant release of fluoride with ACTIVA ${ }^{\mathrm{TM}}$.

However, the results disagreed with Elbahrawy and Attia [15] who found that ACTIVA ${ }^{\mathrm{TM}}$ releases more fluoride than resin-modified glass ionomer. They suggested that this may be due to ACTI$\mathrm{VA}^{\mathrm{TM}}$ composition that contains a patented, resilient resin matrix with energy-absorbing elastomeric components (a blend of diurethane and methacrylates with modified polyacrylic acid and polybutadiene modified diurethane dimethacrylate). This patented resin matrix might affect the permeability of this enhanced RMGI.

Based on the results of the present study, the null hypothesis could be rejected since a statistically significant difference was found in the fluoride, phosphorus, and calcium releasing potentials among the tested materials at each time interval.

\section{Study Limitations}

The present research is in vitro in nature which does not reflect the actual status of fluoride release and antimicrobial properties in the oral cavity.

The follow-up period was short to establish conclusive results regarding the success of both materials. However, this project pro- vides more information and better sight for future longer follow-up trials.

Despite the importance of laboratory studies to answer some questions in a short time, the real performance of restorations can only be determined by long-term clinical trials.

\section{Conclusion}

Based on the previous results, it can be concluded that:

1. Resin modified glass ionomer materials exhibit greater fluoride release than resin-based materials ACTIVIA BioactiveRestorative with the highest fluoride release taking place during the first 24 hours.

2. ACTIVIA Bioactive-Restorative shows a lower fluoride-releasing potential than RMCGIC however, it could provide a relatively constant release throughout 3 months.

3. ACTIVA Bioactive-Restorative provides relatively constant phosphorus release for 3 months, while RMGIC shows lower phosphorus release that increases gradually with time.

4. Calcium release was higher in ACTIVA Bioactive-Restorative compared to RMCGIC.

5. ACTIVA Bioactive-Restorative could be considered a suitable class $\mathrm{V}$ restoration in high caries risk patients.

\section{Conflict of Interest}

The authors declare no conflict of interest.

\section{Bibliography}

1. Bansal R and Bansal A. "Comparative Evaluation of the Amount of Fluoride Release and Re-Release after Recharging from Aesthetic Restorative Materials An In vitro Study". Journal of Clinical and Diagnostic Research 9.8 (2015): ZC11-ZC14.

2. Besinis A., et al. "Review of nanomaterials in dentistry: interactions with the oral microenvironment, clinical applications, hazards, and benefits". ACS nano 9.3 (2015): 2255-2289.

3. Berg JH and Croll TP. "Glass ionomer restorative cement systems an update". International Journal of Paediatric Dentistry 37 (2015): 116-124.

4. Choi Y., et al. "Bioactive solâ€ "gel glass added ionomer cement for the regeneration of tooth structure". Journal of Materials Science 19.10 (2008): 3287. 
5. Croll TP., et al. "Dental Repair Material: A Resin-Modified Glass- Ionomer Bioactive Ionic Resin-Based Composite". Compendium of Continuing Education in Dentistry 36.1 (2015): 60-65.

6. El-Bahrawy EM and Attia RM. "Fluoride Releasing Potential and Recharging Capacity of Different Bioactive Restorative Materials (A Comparative In-Vitro Study)". Egyptian Dental Journal 66.2 (2020): 1295-1309.

7. Garoushi S., et al. "Characterization of fluoride releasing restorative dental materials". Dental Materials Journal 37.2 (2018): 293-300.

8. Gui Y., et al. "Fluoride release and recharge properties of six restorative materials". Chinese Journal of Stomatology 50.1 (2015): 28-32.

9. Karanicolas PJ., et al. "Blinding: Who, what, when, why, how?". Canadian Journal of Surgery 53.5 (2010): 345.

10. Khoroushi M and Keshani F. "A review of glass-ionomers: From conventional glass-ionomer to bioactive glass-ionomer". Dental Research Journal 10.4 (2013): 411-420.

11. Kucukyilmaz E., et al. "Fluoride release/recharging ability and bond strength of glass ionomer cements to sound and caries-affected dentin". Nigerian Journal of Clinical Practice 20.2 (2017): 226-234.

12. Kulkarni PS. "Bioactive Materials: Ion Release, Flexural Strength, Secondary Caries Depth and Elemental Analysis of Surrounding Tooth Structure". Doctoral dissertation, The University of Alabama at Birmingham (2017).

13. Liang K., et al. "Poly (amido amine) and calcium phosphate nanocomposite remineralization of dentin in acidic solution without calcium phosphate ions". Dental Materials 33.7 (2017): 818-829.

14. López-García S., et al. "In vitro Evaluation of the Biological Effects of ACTIVA Kids BioACTIVE Restorative, Ionolux, and Riva Light Cure on Human Dental Pulp Stem Cells". Materials 12.22 (2019): 3694.

15. Morrow B., et al. "Evaluation of $\mathrm{pH}$ Fluoride and Calcium Release for Dental Materials". Journal of Dental Research 96 (2017): 1359.

16. Nagi S M., et al. "Fluoride release and recharge of enhanced resin modi fi ed glass ionomer at different time intervals". Future Dental Journal 4.2 (2018): 221-224.
17. Pameijer CH., et al. "Flexural Strength and Flexural Fatigue Properties of Resin-Modified Glass Ionomers". Journal of Clinical Dentistry 26.1 (2015): 23-27.

18. Prabhakar AR., et al. "Comparative evaluation of the remineralizing effects and surface micro hardness of glass ionomer cements containing bioactive glass (S53P4): an In vitro study". International Journal of Clinical Pediatric Dentistry 3.2 (2010): 69.

19. Quock RL., et al. "Effect of silver diamine fluoride on microtensile bond strength to dentin". Operative Dentistry 37.6 (2012): 610-616.

20. Rodrigues D S., et al. "Mechanical Strength and Wear of Dental Glass-Ionomer and Resin Composites Affected by Porosity and Chemical Composition". Journal of Bio- and Tribo-Corrosion 24.1 (2015): 1-9.

21. Rouessac F and Rouessac A. "Chemical analysis: modern instrumentation methods and techniques". John Wiley and Sons (2013).

22. Sagmak S., et al. "Comparative Evaluation of Antimicrobial Efficacy and Fluoride Release of Seven Different Glass-IonomerBased Restorative Materials". Oral Health and Preventive Dentistry 18.3 (2020): 521-528.

23. Suresh KP. "An overview of randomization techniques: an unbiased assessment of outcome in clinical research". Journal of Human Reproductive Sciences 4.1 (2011): 8.

24. Tiskaya M., et al. "Characterization of the bioactivity of two commercial composites". Dental Materials 35 (2019): 17571768.

25. Zafar MS and Ahmed N. "Therapeutic roles of fluoride released from restorative dental materials". Fluoride 48.3 (2015): 184.

\section{Assets from publication with us}

- Prompt Acknowledgement after receiving the article

- Thorough Double blinded peer review

- Rapid Publication

- Issue of Publication Certificate

- High visibility of your Published work

Website: www.actascientific.com/

Submit Article: www.actascientific.com/submission.php

Email us: editor@actascientific.com

Contact us: +919182824667 